\title{
26 Research Square \\ Caregiver Perspective on The Initial Signs and Symptoms of Metachromatic Leukodystrophy
}

Florian Eichler

Massachusetts General Hospital

Francis Pang

Orchard Therapeutics

Kenneth Howie

Magnolia Innovation

Markus Walz

Magnolia Innovation

Alexandra Wilds

Magnolia Innovation

Carlo Calcagni

Magnolia Innovation

Charlotte Chanson

Orchard Therapeutics

Laura Campbell ( $\square$ laura.campbell@orchard-tx.com )

Orchard Therapeutics https://orcid.org/0000-0003-2593-8006

\section{Research Article}

Keywords: Metachromatic Leukodystrophy, MLD, initial symptoms, disease onset, diagnostic delay, caregiver experience, caregiver language, early-onset, late infantile, juvenile

Posted Date: June 3rd, 2021

DOl: https://doi.org/10.21203/rs.3.rs-546822/v1

License: (c) (1) This work is licensed under a Creative Commons Attribution 4.0 International License.

Read Full License 


\section{Abstract \\ Background}

Metachromatic leukodystrophy (MLD), a relentlessly progressive and ultimately fatal condition, is a rare autosomal recessive lysosomal storage disorder caused by a deficiency of the enzyme arylsulfatase $A$ (ARSA). Historically management has been palliative or supportive care. Hematopoietic stem cell transplantation is poorly effective in early-onset MLD and benefit in late-onset MLD remains controversial. Hematopoietic stem cell gene therapy was recently approved by the European Medicines Agency for early-onset MLD. Treatment benefit is mainly observed at an early disease stage, indicating the need for early diagnosis and intervention. This study contributes a caregiver perspective of initial MLD symptomatology, and thereby aims to improve communication between clinicians and families impacted by this condition and promote a faster path to diagnosis.

\section{Results}

Data was collected through a moderator-assisted online 60-minute survey and 30-minute semi-structured follow-up telephone interview with 21 MLD caregivers in the United States $(n=10)$, the United Kingdom ( $n$ $=5)$, and Germany $(n=6)$. All respondents were primary caregivers of a person with late infantile $(n=13)$, juvenile $(n=7)$ or borderline late infantile/juvenile $(n=1)$ MLD. Caregivers were asked questions related to their child's initial signs and symptoms, time to diagnosis and interactions with healthcare providers. These results highlight the caregiver language used to describe the most common initial symptoms, which can be summarized as developmental stagnation, difficulty walking/running, and clonus/tremors, in the absence of notable prior history. Distinctions between late infantile versus juvenile MLD in symptom onset and disease course were also identified.

\section{Conclusions}

This study captures the most frequent caregiver-reported physical, behavioral, and cognitive signs of MLD leading up to diagnosis. The understanding of the caregiver experience at symptom onset sheds light on a critical window of often missed opportunity for earlier diagnosis and therapeutic intervention in MLD.

\section{Introduction}

Metachromatic leukodystrophy (MLD) is a rare autosomal recessive lysosomal storage disorder caused by mutations in the ARSA gene leading to a deficiency of the enzyme arylsulfatase A (ARSA) [1]. The decreased activity of ARSA results in the accumulation of sulfatides in the central and peripheral nervous systems, leading to microglial damage, neurodegeneration, progressive demyelination, and loss of motor and neurocognitive function [1-4]. Typically, MLD is classified into 3 subtypes by age of onset of first 
symptoms, including late infantile (symptom onset $\leq 30$ months of age), juvenile, further broken out into early juvenile (onset $>30$ months and $<7$ years) and late-juvenile (onset $\geq 7$ years and $<17$ years), and adult (onset $\geq 17$ years). Late infantile and early juvenile MLD together can also be classified more broadly as early-onset MLD (symptoms onset $<7$ years of age) $[3,5,6]$. MLD is characterized by progressive motor and cognitive deterioration [1, 7]. Historical management has been palliative or supportive care. Limited benefit has been observed in the use of hematopoietic stem cell transplantation (HSCT) $[7,8]$. Transplant benefit is only observed in patients whose disease has not significantly progressed, indicating a need for early diagnosis and intervention [9]. Transplantation after the presentation of motor and/or cognitive symptoms significantly limits the chance of the procedure's success and can often result in rapid decline post-transplant [8]. Gene therapy and enzyme replacement therapy are being investigated for the treatment of MLD [10]. Ex vivo hematopoietic stem cell gene therapy was recently approved by the European Medicines Agency.

Early recognition of MLD is crucial to increase the chance of patients qualifying for and benefiting from therapy. However, the initial signs and symptoms can be subtle and non-specific, and patients can go unrecognized for months and years [1]. From a caregiver perspective, this time between symptom onset and diagnosis is characterized by uncertainty, frustration, and fear. While there are a few studies focused on MLD that highlight or include a caregiver perspective, there is continued need to better understand caregivers' experiences early in the disease process to enhance our understanding of how to identify MLD more quickly $[11,12]$.

The goal of this paper is to contribute to our understanding of how caregivers describe the initial symptomatology of MLD and ultimately to improve communication between clinicians and families impacted by this condition. This paper characterizes the initial symptoms recognized by caregivers, perceived speed of disease progression, and time to diagnosis, including possible misdiagnoses.

\section{Methods}

Data were collected through an online survey and qualitative interviews with MLD caregivers in the United States (US), the United Kingdom (UK), and Germany (DE). Data were collected as part of a larger research study on disease burden and quality of life as reported by caregivers of children diagnosed with MLD. The study is managed by Magnolia Innovation on behalf of the study sponsor, Orchard Therapeutics.

\section{Study Design \& Data Collection}

Study protocol and all study material were approved by an external IRB (provider: Pearl IRB, Indianapolis, IN). The survey instrument was designed by Magnolia Innovation and reviewed by clinicians with expert knowledge of MLD, and representatives of patient advocacy groups. Data was collected through moderator-assisted online surveys (length: 60-minutes) and semi-structured telephone interviews (length: 30-minutes). In the survey, caregivers were asked to describe initial symptoms, confirm if and when they sought medical advice after noticing these clues, how much time passed until they sought said advice, 
and at what age their child was diagnosed. During the follow-up interviews, respondents were asked to provide additional details around their interactions with healthcare providers and challenges experienced leading up to diagnosis.

\section{Sample/Respondent Selection}

Respondents were contacted and recruited with the help of patient advocacy groups. During the recruitment process respondents were briefed on study objectives and methodology, answered initial screening questions and provided written consent to participate. While all respondents verbally confirmed the MLD diagnosis during their screening calls, 13 of the 21 respondents also provided additional proof of diagnosis, such as a copy of a clinician's note or genetic testing results. Surveys were completed by 21 respondents from the three countries ( $U S=10, U K=5, D E=6$ ). Nineteen of the 21 respondents participated in follow-up interviews ( 2 declined to participate in the follow-up conversation) and two survey respondents were also joined by their spouse/partner. Survey calls and interviews were conducted in English (for US and UK) and German.

\section{Data Management/Analysis}

Survey data and interview transcripts were further anonymized to blind the sponsor to respondent identity. Survey answers on initial symptoms were coded to identify what type of symptoms were most commonly observed at disease onset. Frequency of codes are reported for total sample and by onset types, for late infantile onset and juvenile onset. Given the small sample size, this study provides descriptive statistics.

Table 1

Patient MLD Onset Type by Country

\begin{tabular}{|llll|}
\hline Country & Total Patients & Late Infantile Onset & Juvenile Onset \\
\hline Patients, n (\%) & $21(100)$ & $13(61.9)$ & $7(33.3)$ \\
\hline US, n (\%)* & $10(47.6)$ & $6(60.0)$ & $3(30.0)$ \\
\hline UK, n (\%) & $5(23.8)$ & $3(60.0)$ & $2(40.0)$ \\
\hline DE, n (\%) & $6(28.6)$ & $4(66.7)$ & $2(33.3)$ \\
\hline $\begin{array}{l}\text { Note: Borderline late infantile/juvenile onset patient case not included in breakout of US sample onset } \\
\text { types. }\end{array}$ & \\
\hline
\end{tabular}

\section{Results}

\section{Respondent Characteristics}

\section{Caregiver Background}


In total, 21 caregivers were included in this study, all of whom were parents of children with MLD.

Seventeen of the 21 caregivers interviewed were mothers (80.9\%), and the remaining caregivers were fathers (one stepfather) of the patients $(4 / 21 ; 19.0 \%)$. Except for one, all children with MLD were alive at the time of research.

\section{Symptom Onset Type Breakout}

Patients were categorized as late infantile MLD if their symptom onset began at, or prior to, 30 months of age. Juvenile MLD categorization was defined by symptom onset between the ages of 31 months and 17 years of age. Thirteen patients (61.9\%) were reported to have late infantile onset and seven patients (33.3\%) were reported as juvenile onset. One caregiver (4.7\%) reported their child received a diagnosis of borderline late infantile/juvenile - this respondent's data was included in qualitative findings, but was excluded from any onset type categorization or statistical analysis. The mean age of these late infantile and juvenile patients at the time of interviews was 5.1 years and 15.6 years, respectively (one late infantile patient had passed away 2 years prior to interview; the patient's age was not represented here, however the patient died at 7.7 years old).

\section{Sample Diagnosis and Treatment}

All patients in the sample (if alive) were diagnosed symptomatically and were at progressed stages of their disease (i.e., severe cognitive impairment and loss of trunk control) at the time of the interview. On average, time between onset of symptoms and caregiver participation in interviews was 5.8 years (range: 1-16.3 years). Four patients in this study received HSCT (19.0\%). (Table 2). 
Table 2

Demographics of Patients as reported by their respective caregiver respondent

$\left.\begin{array}{llll}\text { Characteristics } & \text { Overall }(n= & \text { Late Infantile }(n= & \text { Juvenile }(n \\ & 21) & 13) & 7\end{array}\right)$

Sex

$\begin{array}{llll}\text { Female, } \mathrm{n}(\%) & 14(66.7) & 10(76.9) & 4(57.1) \\ \text { Male, } \mathrm{n}(\%) & 7(33.3) & 3(23.1) & 3(42.9)\end{array}$

Current Age (Years)*

Mean

9.3

5.1

15.6

Median

6.8

4.5

13.1

Range

$3-30.3$

3-11.1

8-30.3

Age at First Symptom Onset (Years)

Mean

3.5

1.5

7.5

Median

1.5

1.5

5.6

Range

$1-14$

$1-2.5$

$4.5-14$

Age at Diagnosis (Years)

Mean

4.7

2.4

8.4

Median

2.8

2.4

6.2

Range

$1.9-14.3$

$1.9-3.5$

5-14.3

Time Between Symptom Onset and Diagnosis (Months)

Mean

14.5

11.2

9.9

Median

10

10

5

Range

$0-90$

2-28

$0-36$

Time Elapsed Since Symptom Onset (Years)

Mean

Median

Range

Note: One child with late infantile onset MLD was not included in age data points as they had passed away. One respondent is not accounted for in onset type breakout due to reportedly being a borderline late infantile/juvenile case. 


\section{Most Common Early Signs of MLD}

Most common initial symptoms reported across all patients were developmental stagnation (38.1\%), difficulty walking/running (38.1\%), and clonus/tremors (28.6\%). Signs of developmental stagnation typically referred to the child not reaching or progressing towards certain expected milestones, such as their child never being able to say more than a few words. Equally common were early signs of difficulty walking/running, such as gait spasticity.

\section{Differences in Initial Symptoms by Age of Onset}

Most common initial symptoms for patients with late infantile MLD were developmental stagnation (53.8\%), difficulty walking/running (30.8\%), and clonus/tremors (30.8\%). In patients with juvenile MLD, change in behavior (57.1\%), difficulties walking/running (57.1\%) and cognitive indications [lack of awareness (42.9\%) and issues with memory (28.6\%)] were most frequently reported (Table 3 ).

Table 3

Caregiver-reported initial symptoms of MLD patients by onset type

\begin{tabular}{|c|c|c|c|}
\hline Initial Symptoms (ordered by total) & Overall $(n=21)$ & Late Infantile $(\mathrm{n}=13)$ & Juvenile $(n=7)$ \\
\hline Developmental Stagnation, n (\%) & $8(38.1)$ & $7(53.8)$ & $1(14.3)$ \\
\hline Difficulty Walking/Running, $\mathrm{n}(\%)$ & $8(38.1)$ & $4(30.8)$ & $4(57.1)$ \\
\hline Clonus/Tremor, n (\%) & $6(28.6)$ & $4(30.8)$ & $1(14.3)$ \\
\hline Change in Behavior/Personality, n (\%) & $5(23.8)$ & $1(7.7)$ & $4(57.1)$ \\
\hline Developmental Delay, n (\%) & $4(19.0)$ & $2(15.4)$ & $1(14.3)$ \\
\hline Lack of Awareness, n (\%) & $3(14.3)$ & $0(0)$ & $3(42.9)$ \\
\hline Coordination/Balance, n (\%) & $2(9.5)$ & $1(7.7)$ & $1(14.3)$ \\
\hline Developmental Regression, $\mathrm{n}(\%)$ & $2(9.5)$ & $1(7.7)$ & $1(14.3)$ \\
\hline Issues with Memory, n (\%) & $2(9.5)$ & $0(0)$ & $2(28.6)$ \\
\hline Learning Difficulties, n (\%) & $2(9.5)$ & $0(0)$ & $2(28.6)$ \\
\hline Visual Impairment, n (\%) & $2(9.5)$ & $2(15.4)$ & $0(0)$ \\
\hline Sleep Issues, n (\%) & $1(4.8)$ & $0(0)$ & $1(14.3)$ \\
\hline Unilateral Muscle Weakness, n (\%) & $1(4.8)$ & $1(7.7)$ & $0(0)$ \\
\hline \multicolumn{4}{|c|}{$\begin{array}{l}\text { Note: Initial symptoms were grouped into buckets based on similarities in response. There is overlap } \\
\text { amongst respondents within each symptom group as many respondents listed more than one initial } \\
\text { symptom. One respondent is not accounted for in onset type breakout due to reportedly being a } \\
\text { borderline late infantile/juvenile case. }\end{array}$} \\
\hline
\end{tabular}

Absence of Causation For Early Signs of MLD 
Caregivers reported an absence of perinatal injury prior to signs of MLD emerging. One caregiver explains, "when she was born, we had a great pregnancy. She was born healthy and she developed normally until 15 months". None of the caregivers had unsolicited reports of pregnancy issues. Caregivers recall little explanation for the onset of symptoms that had first alarmed them. Those who reported developmental stagnation, regression, or delays $(12 / 21,57.1 \%)$ explained how their child was meeting milestones before noticing a disease-related delay or stagnation in their milestones.

\section{Caregiver Language}

\section{Observed Changes in Daily Activities}

Specific terminology or language used by caregivers was captured in detail to see how the early signs of MLD are identified and described by caregivers. (see supplemental materials, Section: "Observed Changes in Daily Activities" for quotes from caregivers). 
Table 4

Language used by caregivers to describe initial signs and symptoms of MLD

\section{Change in Behavior/Personality}

Cried a lot

Getting lost, peeing in pants at school

Not age appropriate behavior

Stubborn

Personality changes, impulsive behavior, loss of interest in activities that he used to be interested in

Clonus/tremor

Stroke-like movement of the arms

Developed a small tremor in her hands

Slight tremor

Slight tremor, eye lid twitching (that Ped. Noticed on regular checkup)

Clonus

Shake really bad after naps, foot tremoring

\section{Coordination/balance}

Uncoordinated, clumsy

Started to lose her balance

Developmental delay

Started walking late as a baby

Developmental delays, cognitive delays

Early to crawl, but late to stand up and holding on to things, gaps between achieving milestones was getting bigger

Late walker

\section{Developmental regression}

Ability to do math, top of his class in 1st grade, 2nd grade couldn't monitor progress and didn't know he was struggling, 3rd grade couldn't add

Regressing

Developmental stagnation

Could never walk independently

Development not progressing 


\section{Change in Behavior/Personality}

Could never run freely

Not progressing with walking (started talking first steps but did not progress after)

She never walked

Wasn't walking, only 6 words

Stagnation in motor development

Never able to ride a bike

Difficulty walking/running

Struggled to run

Wide-legged gait

Could never run freely, twisted foot while running

Walking was stiff, often fell

Striking gait

Trouble walking

Gait spasticity

Unstable walking

Issues with Memory

Forgetful

Peeing pants and forgetting

Lack of Awareness

Getting lost

Lack of concentration, issues with concentration levels

Appearing sleepy and dazed

\section{Learning difficulties}

Ability to do math, top of his class in 1st grade, 2nd grade couldn't monitor progress and didn't know he was struggling, 3rd grade couldn't add

Learning difficulties (learning vocabulary)

\section{Sleep issues}

Issues with sleep 


\section{Change in Behavior/Personality}

\section{Unilateral muscle weakness}

Left was weaker than her right side

\section{Visual impairment}

Sudden squint (18 months)

Went cross-eyed overnight

Changes in behavior, such as inability to perform in school, were cited by 5 caregivers (23.8\%). Another caregiver articulated the changes they noticed in their child with juvenile MLD by recollecting her new difficulties in running errands and changes in behavior, including enuresis.

\section{Peer Comparisons}

Caregivers used peer comparisons to explain the apparent gaps or delays in meeting age-related milestones. As one caregiver put it, their child with late infantile onset MLD was "slower than the average child that we could see" in terms of meeting developmental milestones (see supplemental materials, Section: "Peer Comparisons" for quote from a caregiver).

\section{Describing Symptoms in Caregiver Language}

Caregivers used a variety of terms to describe what are presumably some of the typical early symptoms. For example, caregivers described what is likely clonus as "twitching", "shaking", or "stroke-like movements". To describe difficulty walking, some used more clinical terms, such as "gait spasticity" while others were more general in their descriptions (e.g., "unstable walking"). There was also limited direct use of the words "developmental stagnation" or "developmental delays". Instead, caregivers shared examples of activities that their child was delayed in or unable to achieve, such as "could never run freely". Indications of developmental delays were phrased as milestones that they felt their child was "late" to accomplish such as, "started walking late as a baby" or "late to stand up".

\section{Seeking Medical Consult After First Clues}

\section{Symptoms Triggering Medical Consult}

Gait-related symptoms-including spasticity, inability to walk, slow to walk, or regressing ability to walkwere often triggers to seek medical advice. Over the course of the diagnostic process, caregivers report their children presenting with a constellation of symptoms. Both caregivers and clinicians struggled to put the different signs together, often prolonging the diagnostic journey. As one caregiver recounted, "nobody was putting the jigsaw pieces together and seeing the big picture". Normal pregnancies or absence of birth complications often confounded timely diagnoses, as it was difficult to assess significance of subtle early signs without the ability to attribute to a specific incident. 
After noticing the first concerning clues, $85.7 \%$ (18/21) of caregivers sought medical advice. The mean time to seeking medical consult was 5 weeks (range: 1-24 weeks). Generally, caregivers first visited a pediatrician, family primary care physician (PCP), general practitioner (GP) or (if in the UK) had an inhome health visit, before seeing a specialist. Those that did not immediately seek out medical advice $(14.3 \% ; 3 / 21)$ had waited due to lack of recognition of subtle, gradual symptoms, or misattribution to other confounding factors.

\section{Reasons for Not Seeking Immediate Medical Advice at First Clues}

One respondent's child with late infantile MLD presented with frequent crying, which was difficult to interpret in a 15-month old child. Once they realized that their son had missed the milestone of walking, this prompted them to visit a clinician- this process took around 4 months.

In a different case of a juvenile onset patient, the caregiver clarified that medical advice was not immediately sought as they assumed that the behavioral issues were a sign of puberty. Once gait abnormalities presented, they decided to see a specialist. The time from presentation of behavioral symptoms to specialist visit was approximately 24 weeks.

In a third instance of a juvenile onset patient, the caregiver did not recognize their child's stroke-like arm movement; however, an occupational therapist at the child's school observed the movements, which led to a medical consultation.

\section{Non-Clinical Observers}

Signs and symptoms may first be noted by those involved in childcare, such as teachers and school staff. In an example provided by a caregiver, a nursery teacher's observations prompted a follow-up visit with the GP for a child with juvenile-onset MLD. The initial signs were described by the caregiver as "anomalies", such as concentration issues or non-age-appropriate behavior, until their nursery teacher observed these symptoms and was able to recommend further clinical action (see supplemental materials, Section "Non-Clinical Observers" for quote from a caregiver).

\section{Winding Referral Pathways During Initial Medical Consults}

After initial consultation, caregivers were referred to numerous specialists depending on the presenting symptoms, including endocrinologists, allergists, psychiatrists, physical therapists, neurologists, and geneticists. Ultimately, MLD was reported to be diagnosed by either the neurologist (15/21), geneticist (5/21), or pediatrician (1/21) (see supplemental materials, Section "Referral Pathways During Initial Medical Consults" for quote from a caregiver).

\section{Time to Diagnosis}

\section{Differences in Time to Diagnosis by Onset Type}


Symptom onset for late infantile patients were reported, on average, at 1.5 years old (range: 1.0-2.5 years). Juvenile patients were reported to have symptom onset, on average, at 7.5 years old (range: 5.014.3 years); of these, early juvenile patients reported symptom onset at 5.3 years old $(n=5)$ and late juvenile patients reported first symptoms at 13.0 years old $(n=2)$.

Time from symptom onset to the point of MLD diagnosis was variable, overall averaging 14.5 months. Mean time to diagnosis in late infantile patients was 11.2 months (range: 2-28 months), and 9.9 months (range: 0-36 months) for juvenile patients (Table 2). The time to diagnosis was much longer for one unique borderline late infantile/juvenile case which took up to 90 months to reach a diagnosis from when initial signs were observed (this case was not included in the breakout of time to diagnosis by onset type) (see supplemental materials, Section "Caregiver-Reported Borderline Late Infantile/Juvenile Patient Case" for more information).

\section{Rapid Progression in Time to Diagnosis}

Rapid disease progression is exemplified by one caregiver who shared that their healthy child began experiencing trouble walking at 15 months. From the first signs of disease onset to diagnosis ( 9 months) their child had lost almost all motor function and within the following month had lost all remaining motor function. Nine of the 21 (42.9\%) caregivers reported 4 months or less in time from symptom onset to observed developmental regression in their child.

\section{Misdiagnoses}

\section{Misdiagnoses Received Throughout Diagnostic Journey}

$47.6 \%(10 / 21)$ of caregivers stated that their child was misdiagnosed prior to MLD diagnosis. Occurrences of misdiagnoses were comparable across both late infantile $(46.2 \% ; 6 / 13)$ and juvenile MLD $(42.9 \% ; 3 / 7)$. Misdiagnoses described by respondents encompassed an array of conditions (Table 5); most frequently cited for those with late infantile MLD was 'hypermobility' (33.3\%). 


\section{Misdiagnoses}

\section{Developmental Delay}

Developmental delay/'delayed'

Dyspraxia - developmental coordination disorder/motor learning difficulties

\section{Developmental Disabilities}

Intellectual Disability

Mental Health Disorders

ADHD

Mental Health Issues/Chemical Imbalance

Neurologic/Neuromuscular

Chronic inflammatory demyelinating polyneuropathy (CIDP)

Myasthenia Gravis (MG)

Tethered cord

Transverse myelitis

Other

Growing Pains

Hypermobility
Count of Misdiagnosis

(N)

\section{3}

2

1

1

1

2

1

1

4

1

1

1

1

3

1

2

\section{Role of Caregivers Instincts and Advocacy}

\section{Caregiver Instincts}

Upon presentation of symptoms, caregivers recalled the feelings of first concern for their child. In what may have appeared as subtle and general signs, caregivers described the immediate instincts felt when watching their child's behaviors change or stagnate. One caregiver described it as their "radar went off"these themes of parental instincts and vigilance were often mentioned (see supplemental materials, Section "Caregiver Instincts" for quote from a caregiver).

\section{Caregiver Advocacy for their Child}

Of the 4 caregivers directly asked about their satisfaction during the diagnostic process, 3 (75\%) expressed dissatisfaction and frustrations. One caregiver explained how she felt the need to be her child's own advocate, and said "I was always fighting quite hard, chasing... I just felt like I was fighting all the 
time for clinicians to take me seriously." This caregiver went on to describe the multiple referrals and lack of answers that led her to take matters into her own hands to get a diagnosis of late infantile MLD (see supplemental materials, Section "Caregiver Advocacy for their Child" for quote from a caregiver).

\section{Discussion}

\section{Importance of Early Diagnosis}

This study provides detailed insights into the initial signs and symptoms of MLD in the words of the caregiver. There are few studies that focus specifically on the language used by caregivers of MLD patients-a key information source and important clue for clinicians to address patients' symptoms in a timely manner $[11,12]$. The first signs and symptoms of MLD are an often missed opportunity during the critical window for early therapeutic intervention. Across all patients in this study's sample, time to diagnosis took an average of 14.5 months, which meant that in many cases patients were too far progressed to be suitable candidates for interventional therapies, leaving palliative and supportive care as their only options. As the European Group for Blood and Marrow Transplantation Guidelines suggests, in patients with MLD, HSCT is "recommended in presymptomatic patients or while neuropsychologic function and independence in activities of daily living remain good." [13]. This urgency to treat is exemplified by the limited window before rapid and devastating disease progression occurs. In Kehrer et al. (2011) the time from first motor symptoms to loss of walking without support, the point after which patients experienced rapid disease progression, was only 8 to 27 months for those with late infantile and juvenile onset MLD respectively [14].

There is reasonable opportunity for disease stabilization post-transplant if the patient is presymptomatic or at an early enough stage in their symptom progression particularly in the juvenile form of MLD. The studies conducted by Beschle, et al. (2020), Groeschel, et al. (2016), and van Rappard et al. (2016) emphasize certain prognostic factors attributing to disease stability post-transplant. Baseline characteristics of those who underwent a successful transplant included: presymptomatic patients, acceptable gross motor function levels, low MRI severity scores, among others $[8,15,16]$. Beschle's recent study also explores the short-term effects in an HSCT cohort that did not meet these positive prognostic factors, leading to rapid progression of symptoms at an even faster rate than non-transplanted patients [8]. Similarly, Fumagalli et al. demonstrates how treatment effects of HSC gene therapy were found to be durable and clinically relevant even in early-symptomatic early juvenile patients who were treated prior to moving into the rapidly progressive phase of the disease [17]. The factors associated with an effective and durable response to gene therapy further highlight the need for early disease identification and intervention to give patients the best chance for disease stabilization. Even for patients who are eligible for HSCT, there is a clear need for more effective options given the variability in response. In a single institution cohort of 40 patients who had undergone HSCT, the estimated 6 -year survival was $50 \%$ for late infantile and $59 \%$ for juvenile forms of MLD [9]. Since the late infantile and juvenile forms of MLD account for close to $80 \%$ of all MLD patients, there is a clear underserved population with an urgent need for both earlier recognition and intervention as well as alternatives to HSCT [18]. 


\section{Symptom Onset}

When referring to "first signs and symptoms", caregivers of late infantile MLD most often report "developmental stagnation". This is supported by the Kehrer, et al. (2014), retrospective study on early symptoms of late infantile and juvenile MLD, which reports that about half of the late infantile patients never learned to speak in complete sentences after having acquired one- and two-word sentences within the normal time range, indicating stagnation in language development. Consequently, Kehrer, et al. recommends further investigation if absence of acquisition of complete sentences after initial normal language acquisition is noted [19]. Stagnation is not a well circumscribed symptom, but rather indicates a lack of developmental progress or gaining milestones. Throughout this study, we see this theme of the "persistent toddler" - part of the challenge here is to highlight the lack of developmental progress, with the history of previously normal development as raising a "red flag" and prompting further investigation. Further, the nature of "developmental stagnation" makes it challenging to pinpoint the exact onset of first signs and symptoms. The insidious nature here is worth noting- onset may be a period of concern that develops over time as the stagnation persists rather than a discrete time point. This subtlety is further confounded by the lack of perinatal injury or other clear cause.

\section{Symptom Constellation}

Eichler, et al. (2016) underscores the complex nature of MLD and how initial symptomatology can vary between and within patient types [11]. Our caregiver reports highlight that it is often a constellation of symptoms, rather than an individual symptom, that ultimately leads to diagnosis. These manifestations are a key characteristic of white matter disorders that affect the connecting fibers and thus multiple functions [20]. In our study, $24 \%$ of caregivers recalled both motor (most frequently described as developmental stagnation and/or trouble walking/running) and cognitive symptoms (most frequently described as development delays and changes in behavior) developing at disease onset. Fumagalli, et al. (2021) also underlined the sequence of symptoms that develop at disease onset. For instance, in early juvenile patients, they found that even those who first presented with isolated behavioral or cognitive impairment, motor symptoms occurred within the next few months. [3]. While a focus upon multiple symptoms rather than individual ones may unfortunately warrant passing of time as further symptoms present, it is an important characteristic of the disease. A confounding factor is the variability and nonspecific nature of these earliest signs and symptoms that can add to the challenge of connecting these constellations of symptoms to MLD specifically. Nonetheless, recognizing the common language used by caregivers to describe the early constellation of symptoms can be another step towards earlier diagnosis.

\section{Late Infantile versus Juvenile MLD}

Kehrer et al. (2020) found that all patients with late infantile and early juvenile onset MLD presented with motor symptoms (with or without additional cognitive symptoms). This study of 97 patients highlights that a small subset $(3 / 35,9 \%)$ of late infantile patients will present with both motor and cognitive symptoms, while most do present with motor symptoms only. Isolated cognitive symptoms were found to occur only in older children and adults $-39 \%$ of early-juvenile patients presented with both motor and 
cognitive symptoms as opposed to $61 \%$ of the late-juvenile cohort who exhibited isolated cognitive symptoms at disease onset [21]. Similarly, our study found that a majority of late infantile patients present with motor symptoms and directionally less recognition of cognitive changes compared to juvenile cases. These distinctions may be more dependent on where these children are on the curve of motor and intellectual developmental milestones at the time of disease progression rather than any fundamental differences in the pathology of the disease. Changes in personality or behavior, for instance, are more noticeable in an older child at that stage of development as opposed to an infant where these cognitive signs may be less apparent. In juvenile cases, this clearer behavioral symptomology that can be distinguished from their normal behaviors and performance provides a more concrete picture in terms of identifying regression. Conversely, in late infantile cases, the stagnation, or absence of development, often reported can lack specificity and be more difficult to translate into a definitive sign of MLD.

\section{Absence of Notable Prior History}

Confounding diagnosis is the lack of predicated indications of concerning signs in these patients. All the above points gain further importance in light of normal pregnancy and birth history, creating a discrepancy and lack of explanation for the initial presenting symptoms. The absence of notable prior history becomes an important clue to diagnosis and a vital part of the full picture in assessing the first signs and symptoms of MLD.

\section{Call to Action}

The findings supported by this research provide a clear call to action for clinicians across specialties to support broader awareness of MLD and its early indicators as described by caregivers. It is important for the medical community to recognize caregiver-reported symptoms consistent with MLD to direct patients to immediate appropriate testing $[22,23]$. Understanding the language used can be educational for caregivers, clinicians, and patient advocacy communities to keep an open and understanding dialogue around this condition and its related early manifestations. Increasingly critical assessment of these early signs can facilitate more rapid referral to proper specialists and streamline the referral pathway [18]. There is a recognizable opportunity for success that comes from providers taking "ownership" of the case as well. A challenge in rare diseases is that due to their specialized nature referrals are often thought to be the necessary and limiting factor. However, "referral" does not exclude taking "ownership" - the nonspecialized nurse, for example, can refer while also pushing the PCP to initiate brain imaging.

It is also possible that other non-clinical persons, such as teachers and school behavioral specialists, pick up on these signs and escalate concerns as necessary to parents or clinicians. Those involved in the social sphere of interactions with these families may be well suited to connect patients with the medical community, should their awareness of the neurological signs and symptoms be improved.

While this study does support means by which to help clinicians observe and therefore diagnose MLD earlier, we acknowledge that MLD is a rare condition with symptoms that are difficult to identify. It is unlikely, even with concrete anecdotes and language to listen for, that every general practitioner will be 
able to discern these subtle signs and make the connection to a leukodystrophy. Ultimately, newborn screening (NBS) will be crucial to diagnosing MLD as early as possible. NBS has precedent in other lysosomal storage disorders, such as MPS-1 where windows of therapeutic intervention are also best when performed before significant disease progression [24]. The development of MLD treatments recently approved, and on the horizon, also further highlights the need for NBS to ensure early patient identification and optimal therapeutic benefit.

\section{Limitations}

It is important to note the potential limitations of the data as they are presented here. The respondents participating in this study are restricted to members of a convenience sample, such that their experiences and perspectives may differ from those of the real-world population of interest. These respondents were also asked to use their recollection rather than medical records for open-ended questions. This may have resulted in variability, recall bias, and inaccuracy of certain data. Given the small sample size, geographical generalizability and comparison across counties can be limited. However, relevance to caregivers and clinicians as a whole is not expected to be limited. However, due to the rarity of the disease this study highlights the importance of the caregiver experience that will enhance the body of literature for MLD recognition and treatment.

\section{Conclusion}

This study highlights the most frequently reported signs of MLD leading up to diagnosis as described by caregivers. The findings from this study offer insight into the key red flags healthcare providers should be cognizant of in dialogue with caregivers that can be suggestive of MLD, including the constellation of symptoms coupled with lack of notable prior history. However, the variability and lack of specificity in symptoms remain a confounding factor in making the accurate and more immediate connection to MLD. Broadening disease awareness by capturing these detailed cases of caregiver language used to describe the early indicators of MLD is crucial to ensure rapid testing and diagnosis of diseases such as MLD that are more easily treatable in the early stages. The findings supported by this research provide a clear call to action for clinicians across specialties to drive quicker attention to the first caregiver-reported signs to promote early identification of MLD as well as earlier therapeutic intervention and improved outcomes.

\section{Declarations}

\section{Ethics approval and consent to participate}

- All participants consented to their anonymized data, including anonymized quotes and pseudonymized and/or aggregated information to be used for this research.

- Study protocol and all study material were approved by an external Institutional Review Board (provider: Pearl IRB, Indianapolis, IN). 
- Not applicable.

\section{Availability of data and materials}

- The datasets generated and analyzed during this study are not publicly available due to individual patient privacy protections.

\section{Competing interests}

- Data presented in this paper was collected as part of a study sponsored and funded by Orchard Therapeutics, Ltd., London, UK and was designed through a collaboration among the sponsor and Magnolia Innovation, LLC, Hoboken, NJ.

- Data was collected by Magnolia Innovation.

- Dr. Eichler has previously served on an advisory board for Orchard Therapeutics but has no current conflicts of interest with Orchard nor Magnolia Innovation.

- Mr. Pang, Ms. Chanson, and Dr. Campbell are previous or current employees of sponsor, Orchard Therapeutics, Ltd.

- Mr. Calcagni, Mr. Howie, Mr. Walz, and Ms. Wilds are previous or current employees of Magnolia Innovation, LLC.

\section{Funding}

- This research did not receive any specific grant from funding agencies in the public, commercial, or not-for-profit sectors

\section{Authors' contributions}

- All authors have made substantial contributions to the concept or design of the work or acquisition, analysis, or interpretation of data. All authors read and approved the final manuscript.

\section{Acknowledgements}

- The researchers would like to thank the study respondents and their families as well as the patient advocacy organizations for their support including: MLD Foundation, MLD Support Association UK, and ELA Deutschland e.V.

- The authors gratefully acknowledge the contribution of the caregivers who participated in the study and their families.

\section{References}

1. van Rappard DF, Boelens JJ, Wolf NI. Metachromatic leukodystrophy: Disease spectrum and approaches for treatment. Best Pract Res Clin Endocrinol Metab. 2015;29(2):261-73. doi:10.1016/j.beem.2014.10.001. 
2. Gieselmann V, Krägeloh-Mann I. Metachromatic leukodystrophy-an update. Neuropediatrics. 2010;41(1):1-6. doi:10.1055/s-0030-1253412.

3. Fumagalli F, Zambon AA, Rancoita P, Baldoli C, Canale S, Spiga I, Medaglini S, Penati R, Facchini M, Ciotti F, Sarzana M, Lorioli L, Cesani M, Sora M, Del Carro U, Cugnata F, Antonioli G, Recupero S, Calbi V, Di Serio C, ... Sessa M. Metachromatic Leukodystrophy: A Single-Centre Longitudinal Study Of 45 Patients. J Inherit Metab Dis. 2021. 10.1002/jimd.12388. Advance online publication. https://doi.org/10.1002/jimd.12388.

4. Lattanzi A, Neri M, Maderna $C$, et al. Widespread enzymatic correction of CNS tissues by a single intracerebral injection of therapeutic lentiviral vector in leukodystrophy mouse models. Hum Mol Genet. 2010;19(11):2208-27. doi:10.1093/hmg/ddq099.

5. Sessa M, Lorioli L, Fumagalli F, et al. Lentiviral haemopoietic stem-cell gene therapy in early-onset metachromatic leukodystrophy: an ad-hoc analysis of a non-randomised, open-label, phase 1/2 trial. Lancet. 2016;388(10043):476-87. doi:10.1016/S0140-6736(16)30374-9.

6. Mahmood A, Berry J, Wenger DA, et al. Metachromatic leukodystrophy: a case of triplets with the late infantile variant and a systematic review of the literature. J Child Neurol. 2010;25(5):572-80. doi:10.1177/0883073809341669.

7. Kohlschütter A. Lysosomal leukodystrophies: Krabbe disease and metachromatic leukodystrophy. Handb Clin Neurol. 2013;113:1611-8. doi:10.1016/B978-0-444-59565-2.00029-0.

8. Beschle J, Döring M, Kehrer C, et al. Early clinical course after hematopoietic stem cell transplantation in children with juvenile metachromatic leukodystrophy. Mol Cell Pediatr. 2020;7(1):12. Published 2020 Sep 3. doi:10.1186/s40348-020-00103-7.

9. Boucher AA, Miller W, Shanley R, et al. Long-term outcomes after allogeneic hematopoietic stem cell transplantation for metachromatic leukodystrophy: the largest single-institution cohort report. Orphanet J Rare Dis. 2015;10:94. doi:10.1186/s13023-015-0313-y. Published 2015 Aug 7.

10. Patil SA, Maegawa GH. Developing therapeutic approaches for metachromatic leukodystrophy. Drug Des Devel Ther. 2013;7:729-45. doi:10.2147/DDDT.S15467. Published 2013 Aug 8.

11. Eichler FS, Cox TM, Crombez E, Dali C, Kohlschütter A. Metachromatic Leukodystrophy: An Assessment of Disease Burden. J Child Neurol. 2016;31(13):1457-63. doi:10.1177/0883073816656401.

12. Harrington $M$, Whalley $D$, Twiss $\mathrm{J}$, et al. Insights into the natural history of metachromatic leukodystrophy from interviews with caregivers. Orphanet J Rare Dis. 2019;14(1):89. Published 2019 Apr 29. doi:10.1186/s13023-019-1060-2.

13. Peters C, Steward CG, National Marrow Donor Program; International Bone Marrow Transplant Registry; Working Party on Inborn Errors, European Bone Marrow Transplant Group. Hematopoietic cell transplantation for inherited metabolic diseases: an overview of outcomes and practice guidelines. Bone Marrow Transplant. 2003;31(4):229-39. doi:10.1038/sj.bmt.1703839.

14. Kehrer C, Blumenstock G, Gieselmann V, Krägeloh-Mann I, GERMAN LEUKONET. The natural course of gross motor deterioration in metachromatic leukodystrophy. Dev Med Child Neurol. 
2011;53(9):850-5. doi:10.1111/j.1469-8749.2011.04028.x.

15. Groeschel S, Kühl JS, Bley AE, et al. Long-term Outcome of Allogeneic Hematopoietic Stem Cell Transplantation in Patients With Juvenile Metachromatic Leukodystrophy Compared With Nontransplanted Control Patients. JAMA Neurol. 2016;73(9):1133-40. doi:10.1001/jamaneurol.2016.2067.

16. van Rappard DF, Boelens JJ, van Egmond ME, et al. Efficacy of hematopoietic cell transplantation in metachromatic leukodystrophy: the Dutch experience. Blood. 2016;127(24):3098-101. doi:10.1182/blood-2016-03-708479.

17. Francesca Fumagalli V, Calbi M, Sessa, et al. Lentiviral hematopoietic stem and progenitor cell gene therapy provides durable clinical benefit in early-symptomatic early-juvenile metachromatic leukodystrophy, Molecular Genetics and Metabolism, Volume 132, Issue 2, 2021, Page S40. https://doi.org/10.1016/j.ymgme.2020.12.081.

18. Brown TM, Martin S, Fehnel SE, Deal LS. Development of the Impact of Juvenile Metachromatic Leukodystrophy on Physical Activities scale. J Patient Rep Outcomes. 2017;2(1):15. doi:10.1186/s41687-018-0041-x.

19. Kehrer C, Groeschel S, Kustermann-Kuhn B, et al. Language and cognition in children with metachromatic leukodystrophy: onset and natural course in a nationwide cohort. Orphanet J Rare Dis. 2014;9:18. Published 2014 Feb 5. doi:10.1186/1750-1172-9-18.

20. Schmahmann JD, Smith EE, Eichler FS, Filley CM. Cerebral white matter: neuroanatomy, clinical neurology, and neurobehavioral correlates. Ann N Y Acad Sci. 2008;1142:266-309. doi:10.1196/annals.1444.017.

21. Kehrer $C$, Elgün $S$, Raabe $C$, et al. Association of age at onset and first symptoms with disease progression in patients with metachromatic leukodystrophy [published online ahead of print, 2020 Oct 12]. Neurology. 2020. 10.1212/WNL.0000000000011047. doi:10.1212/WNL.0000000000011047.

22. Cardinali P, Migliorini L, Rania N. The Caregiving Experiences of Fathers and Mothers of Children With Rare Diseases in Italy: Challenges and Social Support Perceptions. Front Psychol. 2019;10:1780. Published 2019 Aug 5. doi:10.3389/fpsyg.2019.01780.

23. Bronstein MG, Kakkis ED. Patients as key partners in rare disease drug development. Nat Rev Drug Discov. 2016;15(11):731-2. doi:10.1038/nrd.2016.133.

24. Schielen PCJI, Kemper EA, Gelb MH. Newborn Screening for Lysosomal Storage Diseases: A Concise Review of the Literature on Screening Methods, Therapeutic Possibilities and Regional Programs. Int J Neonatal Screen. 2017;3(2):6. doi:10.3390/ijns3020006.

\section{Supplementary Files}

This is a list of supplementary files associated with this preprint. Click to download. 
- AdditionalFilelnitialSignsandSymptomsofMLD18May2021.pptx 